\title{
10-Gb/s Upgrade of Bidirectional CWDM Systems Using Electronic Equalization and FEC
}

\author{
P. J. Winzer, Member, IEEE, Member, OSA, F. Fidler, M. J. Matthews, L. E. Nelson, H. J. Thiele, J. H. Sinsky, \\ S. Chandrasekhar, Fellow, IEEE, M. Winter, D. Castagnozzi, L. W. Stulz, and L. L. Buhl
}

\begin{abstract}
We discuss options for upgrading coarse wavelength-division multiplexed (CWDM) optical access links over standard single-mode fiber (SSMF) by increasing per-channel data rates from 2.5 to $10 \mathrm{~Gb} / \mathrm{s}$. We identify electronic equalization and forward error correction (FEC) as the enabling technologies to overcome the dispersion limit of SSMF. In addition, we show how FEC enhances the tolerance to in-band crosstalk, and paves the way toward fully bidirectional CWDM transmission. Due to the lack of CWDM sources rated for $10-\mathrm{Gb} / \mathrm{s}$ operation, we demonstrate full-spectrum (1310 to $1610 \mathrm{~nm})$ 10-Gb/s CWDM transmission over standard-dispersion fiber using uncooled, directly modulated lasers specified for $2.5 \mathrm{~Gb} / \mathrm{s}$. All $16 \mathrm{CWDM}$ channels could be transmitted over more than $40 \mathrm{~km}$, yielding a capacity-times-distance product of $6.4 \mathrm{~Tb} / \mathrm{s} / \mathrm{km}$. The longest transmission distance $(80 \mathrm{~km})$ was achieved at $1610 \mathrm{~nm}$, equivalent to $1600 \mathrm{ps} / \mathrm{nm}$ of chromatic dispersion.
\end{abstract}

Index Terms-Crosstalk, dispersion, equalizer, forward error correction (FEC), wavelength-division multiplexing (WDM).

\section{INTRODUCTION}

$\mathbf{C}$ OARSE wavelength-division multiplexing (CWDM) is becoming increasingly important for fiber-exhaust optical access applications like metro-feeders, central-office interconnects, storage-area networks (SANs), and passive WDM optical networks (WDM-PONs). By using inexpensive components, such as uncooled directly modulated lasers (DMLs) and thin-film multiplexing filters, CWDM systems allow for the low-cost deployment of short-reach links with appreciable capacity. At present, ITU standards G.694.2 and G.695 specify full-spectrum CWDM systems with up to 18 wavelength channels, 20-nm spaced between 1271 and 1611 $\mathrm{nm}$, with per-channel bit rates up to $2.5 \mathrm{~Gb} / \mathrm{s}$ and reach up to some $80 \mathrm{~km}$ of standard single-mode fiber (SSMF). Bidirectional transmission over a single fiber is accomplished by the use of separate wavelengths for each direction. In order to utilize the entire 340-nm wide CWDM band, low water-peak single-mode fiber (LWPF) has to be used. This fiber type, which is increasingly becoming an alternative to SSMF, does

Manuscript received June 28, 2004.

P. J. Winzer, M. J. Matthews, J. H. Sinsky, L. W. Stulz, and L. L. Buhl are with the Bell Laboratories, Lucent Technologies, Holmdel, NJ 07733 USA (e-mail: peter.winzer@ieee.org).

F. Fidler is with the Institute of Communications and Radio-Frequency Engineering, Vienna University of Technology, Vienna, Austria.

H. J. Thiele was with the OFS, Somerset, NJ 08873 USA. He is now with Siemens, 81379 Munich, Germany.

L. E. Nelson is with the OFS, Somerset, NJ 08873 USA.

M. Winter and D. Castagnozzi are with the AMCC, Andover, MA 01810 USA.

Digital Object Identifier 10.1109/JLT.2004.840369

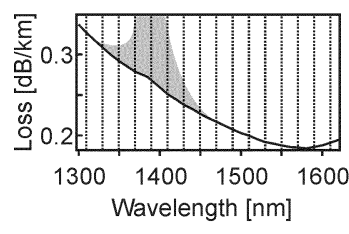

(a) (b)

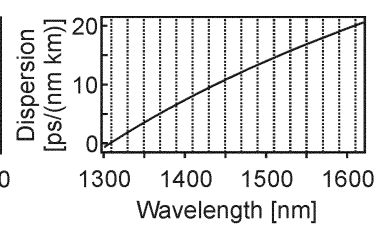

Fig. 1. Attenuation (a) and chromatic dispersion (b) of standard-dispersion low water-peak fiber (LWPF). Shaded area: Additional attenuation due to the water peak of legacy SSMF. Grid lines indicate CWDM channel wavelengths.

not exhibit the typical $\mathrm{OH}^{-}$-absorption peak, which for legacy SSMF is centered at $1385 \mathrm{~nm}$ and inhibits transmission of up to four CWDM channels. Otherwise, standard LWPF has the same properties as SSMF, in particular the same chromatic dispersion characteristics. Fig. 1(a) and (b) shows attenuation and dispersion of AllWave fiber, which is the LWPF used in our experiments. The grid lines indicate the CWDM channel wavelengths. The shaded region in Fig. 1(a) represents the attenuation due to the water-peak of legacy SSMF.

Previous CWDM experiments have demonstrated full-band transmission up to $75 \mathrm{~km}$ of standard-dispersion LWPF [1], using 16 DMLs operating at $2.5 \mathrm{~Gb} / \mathrm{s}$, and resulting in an aggregate system capacity of $40 \mathrm{~Gb} / \mathrm{s}$. Further increasing capacity without resorting to more complex dense WDM (DWDM) overlays [2] naturally asks for the migration to higher per-channel CWDM data rates. This has pushed the design and data modulation of uncooled DMLs to $10 \mathrm{~Gb} / \mathrm{s}$ [3]-[5] and beyond [6], [7]. However, in addition to the difficulties of manufacturing low-cost yet high-speed laser sources, the high frequency-chirp inherent to DMLs poses significant problems in upgrading CWDM systems to $10-\mathrm{Gb} / \mathrm{s}$ line rates. Due to the high dispersion of standard fiber [zero-dispersion wavelength $\left(\lambda_{0}\right)$ at $1310 \mathrm{~nm}, 17 \mathrm{ps} /(\mathrm{nm} \mathrm{km})$ dispersion at $1550 \mathrm{~nm}$, cf. Fig. 1(b)], typically no more than 3 to $10 \mathrm{~km}$ can be bridged at $10 \mathrm{~Gb} / \mathrm{s}$ without the use of additional dispersion-mitigating strategies. Several ways of overcoming the dispersion problem at $10 \mathrm{~Gb} / \mathrm{s}$ have been proposed for CWDM, including nonzero dispersion-shifted fiber (NZDF) [3], [8], negative-dispersion fiber [9], and electronic equalization together with forward error correction (FEC) [10]. Solutions along these lines have recently been reported for the related class of DWDM metropolitan area systems operating around $1550 \mathrm{~nm}$ as well [11]-[13].

In this paper, we report on record CWDM transmission capacities based on potentially low-cost and highly integrated electronic equalization in combination with FEC. Throughout our experiments, we exclusively used commercially available CWDM components, such as 16-channel CWDM thin-film 


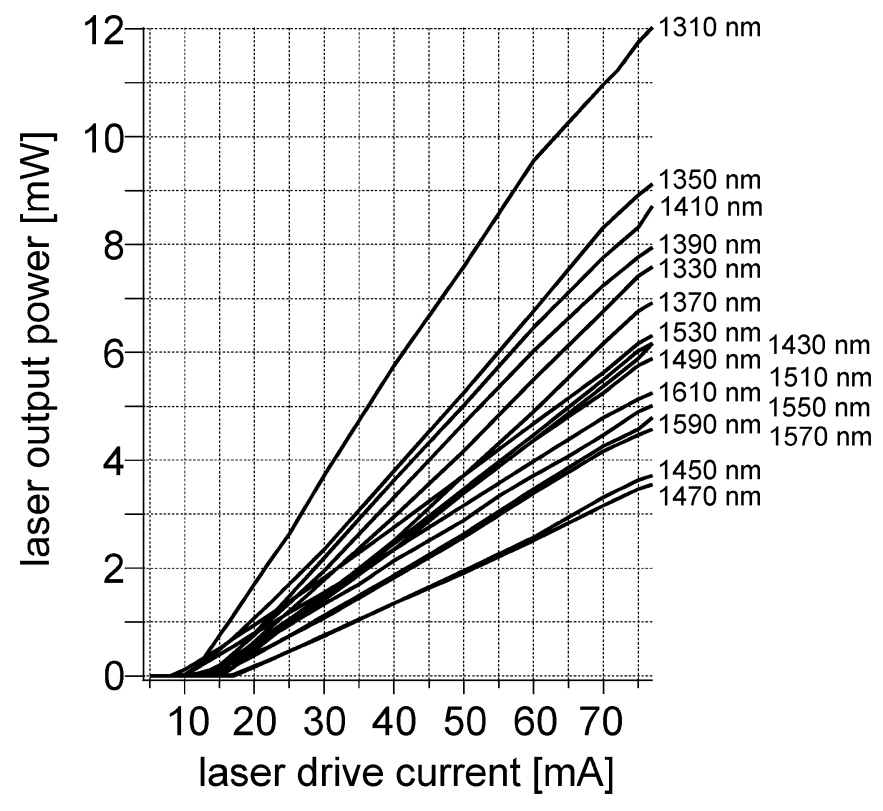

Fig. 2. Widely varying static characteristics of the nonselected $2.5-\mathrm{Gb} / \mathrm{s}$ DMLs, covering the full 16-channel CWDM band from 1310 to $1610 \mathrm{~nm}$.

multiplexers, standard-dispersion low water-peak fiber (cf. Fig. 1), and 16 uncooled DMLs to cover the CWDM band from 1310 to $1610 \mathrm{~nm}$. Due to the unavailability of a full set of $10-\mathrm{Gb} / \mathrm{s}$ rated lasers across the entire CWDM band, we used 2.5-Gb/s rated DMLs. We expect future systems employing $10-\mathrm{Gb} / \mathrm{s}$ rated sources to outperform our results. In our experiments, highly integrated electronic equalization was employed to combat the limited modulation bandwidth of our laser sources as well as chromatic dispersion for the longer wavelength channels. The use of FEC established higher system margins and also increased the tolerance to in-band crosstalk, thus enabling fully bidirectional CWDM transmission.

This paper is structured as follows: Section II discusses the laser sources used in our experiments. Section III describes the employed electronic equalization and FEC. Section IV deals with the improvement in receiver performance and dispersion tolerance from the use of electronic equalization, and Section $\mathrm{V}$ describes how FEC allows for higher amounts of in-band crosstalk in a fully bidirectional system. Section VI then describes full-spectrum system experiments, using all ingredients developed in Sections II-V.

\section{CWDM LASER SOURCES}

In order to demonstrate full-spectrum 10-Gb/s CWDM transmission despite the unavailability of $10-\mathrm{Gb} / \mathrm{s}$ rated laser sources across the entire CWDM band, we employed 16 commercially available, uncooled DMLs rated for $2.5-\mathrm{Gb} / \mathrm{s}$ operation, but modulated them at $10-\mathrm{Gb} / \mathrm{s}$ data rates [8]. Note that our DMLs were not preselected, and thus had widely varying static as well as dynamic characteristics. Fig. 2 shows the output power versus drive current characteristics of all 16 lasers, showing widely varying thresholds $(8-17 \mathrm{~mA})$ and slope efficiencies (0.06-0.2 $\mathrm{mW} / \mathrm{mA})$.

To enable operation of our 2.5-Gb/s-rated DMLs far beyond their specified bandwidth, we mounted each laser at the end

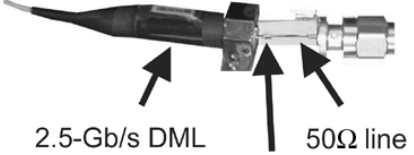

$47 \Omega$ chip resistor

Fig. 3. To improve modulation performance, each laser in a coaxial package was mounted at the end of a $50-\Omega$ microstrip line, in series with a $47-\Omega$ surface mount chip resistor.

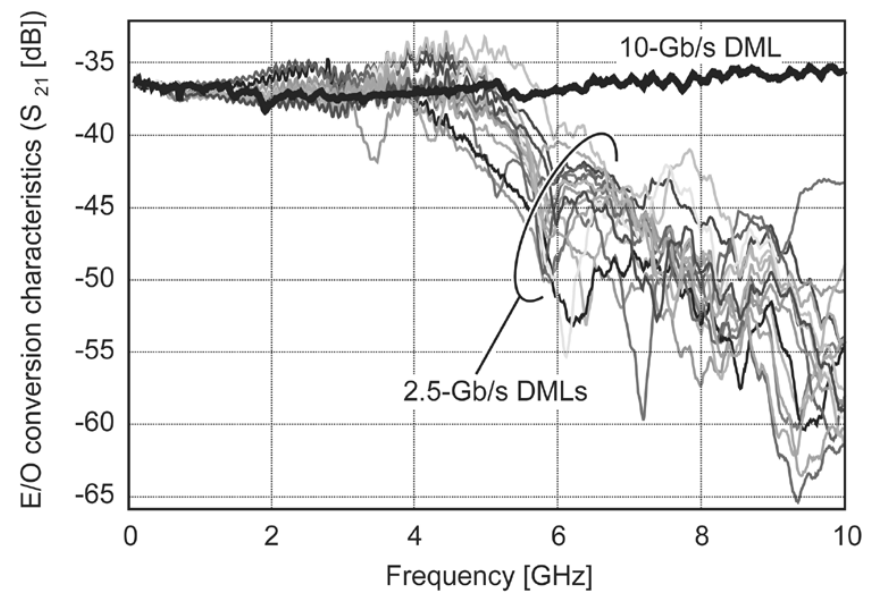

Fig. 4. Modulation characteristics of the 2.5-Gb/s DMLs using the improved mounting of Fig. 3. The characteristics of a $10-\mathrm{Gb} / \mathrm{s}$ rated DML is shown for reference.

of a $50-\Omega$ microstrip line, in series with a $47-\Omega$ surface mount chip resistor placed as close to the laser's coaxial package as possible (see Fig. 3). This careful laser mounting significantly flattened the DMLs' modulation response across the data bandwidth $(<4 \mathrm{GHz})$ despite lowering the overall modulation efficiency. The mounting had no impact on the high-frequency roll-off, which was mostly determined by the intrinsic properties of the laser chips themselves. Fig. 4 shows the small-signal modulation characteristics of all $16 \mathrm{DMLs}$ at $25^{\circ} \mathrm{C}$, together with the characteristics of a commercial 10-Gb/s-rated DML at $1550 \mathrm{~nm}$. At frequencies below $4 \mathrm{GHz}$, both the $2.5-\mathrm{Gb} / \mathrm{s}$ DMLs and the $10-\mathrm{Gb} / \mathrm{s}$ DML exhibit similar performance. A fast roll-off at about $4 \mathrm{GHz}$ is consistently found for all $2.5-\mathrm{Gb} / \mathrm{s}$ DMLs. Apart from the significant scatter in the lasers' frequency response, we also noticed that for similar bias currents some DMLs yielded better results when driven with low data signal amplitudes, leading to extinction ratios of only 3-4 dB [6]-[8], while others preferred to be driven with higher drive amplitudes. For the latter, the '0'-level came close to the lasing threshold, resulting in better extinction ratios at the expense of significant amplitude overshoot. Fig. 5 shows the back-to-back eye diagrams obtained for all 16 lasers when optimizing the driving conditions for maximum transmission distance (see Section VI).

Fig. 6 shows the back-to-back sensitivities (circles) using a standard $10-\mathrm{Gb} / \mathrm{s}$ pin-type photoreceiver without electronic equalization. All 16 DMLs were driven with an FEC-precoded pseudorandom bit sequence (PRBS) of length $2^{31}-1$ using nonreturn-to-zero modulation at $10.664 \mathrm{~Gb} / \mathrm{s}$. This data rate corresponds to a $9.953-\mathrm{Gb} / \mathrm{s}$ OC-192 information bit rate and a $7.14 \%$ FEC overhead (see Section III). The sensitivities are 


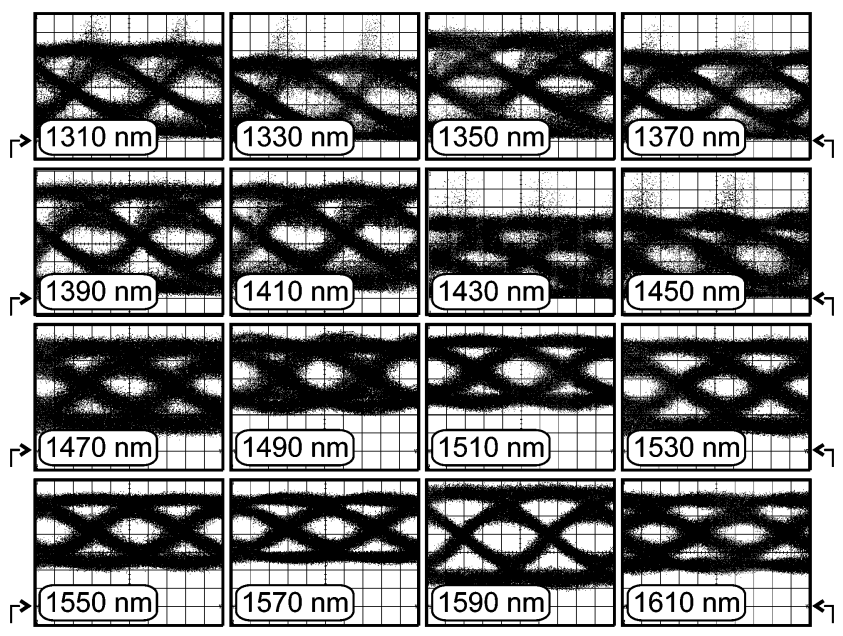

Fig. 5. Back-to-back eye diagrams of all 16 DMLs. Drive conditions were optimized for maximum transmission distance (cf. Section VI).

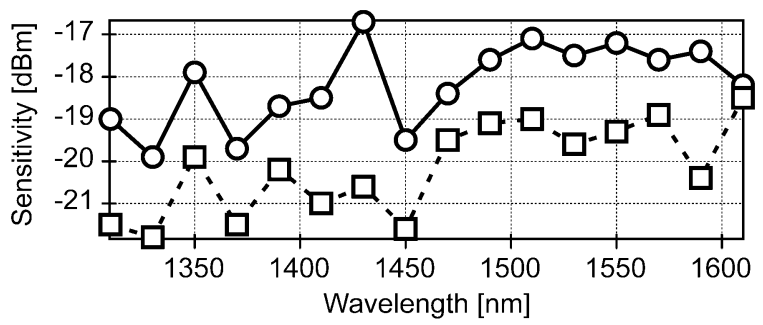

Fig. 6. Back-to-back sensitivities of all $16 \mathrm{CWDM}$ channels (channel BER $=$ $10^{-3}$; corrected BER $\left.<10^{-15}\right)$. Drive conditions optimized for maximum transmission distance (cf. Section VI). Circles: without equalization. Squares: with equalization (cf. Section III).

taken at a channel bit-error ratio (BER) of $10^{-3}$, yielding a corrected (post-FEC) BER of better than $10^{-15}$. Note that the CMOS-based FEC board (cf. Section III) was included in all our BER measurements. This way, we were sure to fully include all deteriorating effects of burst errors on FEC performance in our measurement results. The significant (3-dB) spread in receiver sensitivities seen in Fig. 6 further reflects the nonselected nature of our 2.5-Gb/s DML sources. Expectedly, the spread in receiver sensitivity is lower at the $10^{-3}$ channel BER enabled by the use of FEC than it was in an earlier experiment at BER $=10^{-9}$ that used no coding [8]. In that experiment, we demonstrated unequalized $10-\mathrm{Gb} / \mathrm{s}$ CWDM transmission over $40 \mathrm{~km}$ of non-dispersion-shifted LWPF without FEC, and measured up to $8 \mathrm{~dB}$ sensitivity variation across the CWDM band at $\mathrm{BER}=10^{-9}$, but no indication of an error floor down to $\mathrm{BER}=10^{-10}$. It is also worth mentioning at this point that we tested our lasers at elevated temperatures, where DML characteristics tend to rapidly degrade [5]. Case temperatures of more than $65^{\circ} \mathrm{C}$ were well supported by all 16 DMLs [8].

\section{ELECTRONIC EQUALIZATION AND FEC}

The chipset for electronic equalization and FEC [14] consisted of a CMOS-based FEC device and a SiGe-based, highly integrated equalization circuit, as shown in Fig. 7.

The equalization chipset incorporates a combination of several previously studied equalization techniques [15]-[18], such

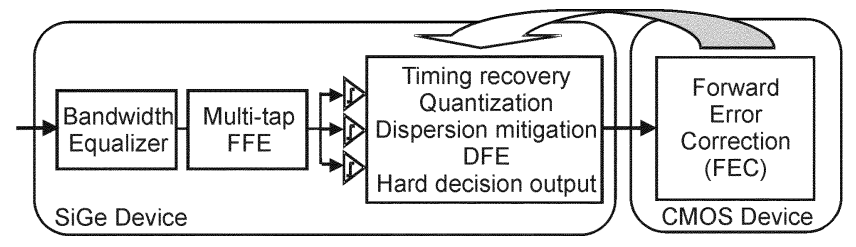

Fig. 7. Block diagram of the integrated electronic equalization and FEC chip set used in the equalized receiver.

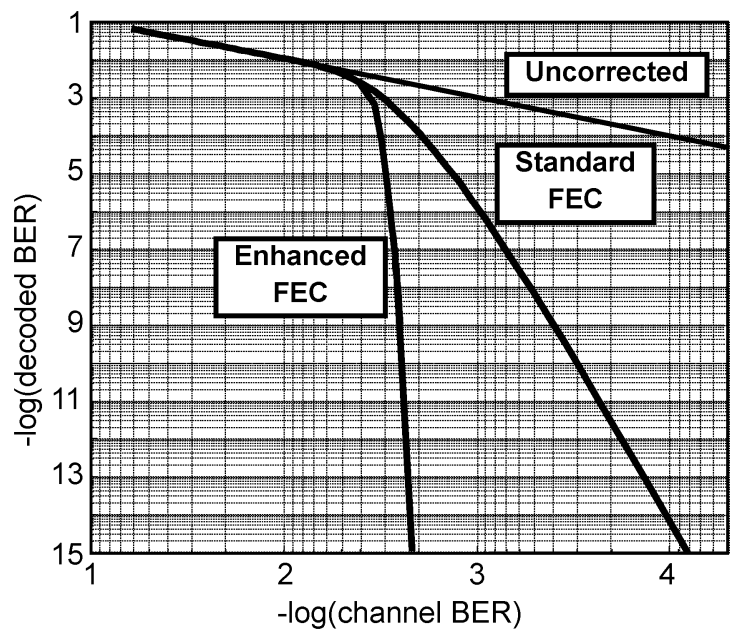

Fig. 8. Correction curves of the enhanced FEC scheme used in our experiment. For comparison, standard RS $(255,239)$ FEC and the FEC-free (uncorrected) case are also shown.

as adaptive thresholding, feed forward equalization (FFE), decision feedback equalization (DFE), and a reduced-complexity version of maximum likelihood sequence estimation (MLSE). The signal is first passed through a variable-gain stage, a variable peaking circuit for bandwidth equalization, and a multitap FFE. A DFE in combination with multiple adaptive thresholding provides quantized information for subsequent soft-decision decoding by simplified, MLSE-like processing and FEC feedback. Full clock and data recovery as well as SFI-4 compliant demultiplexing are part of the chipset. A parallel subrate feedback channel between the SiGe device and the FEC chip provides rapid automatic equalizer adaptation over a broad range of signal conditions.

Fig. 6 (squares) shows the back-to-back sensitivities of the 16 CWDM channels using electronic equalization in combination with the same photoreceiver front-end that was used for the measurements without equalization (circles). Note that the equalizer is able to improve the back-to-back sensitivities of our limited-bandwidth DMLs by up to $4 \mathrm{~dB}$.

The implemented FEC scheme has a $7.14 \%$ bit rate overhead including framing, and complies with ITU standard G.709. The FEC is built on interleaved Reed-Solomon (RS) and Bose-Chandhuri-Hocquenghem (BCH) codes, and for additive Gaussian noise is able to correct channel BERs of $<2 \cdot 10^{-3}$ to values $<10^{-15}$, as visualized in the BER conversion curves shown in Fig. 8 [14]. For comparison, we also show the uncorrected (FEC-free) case, as well as the BER conversion curve for the standard RS $(255,239)$ FEC as specified in ITU G.709, with a channel BER requirement of $6 \cdot 10^{-5}$ for an output BER of less than $10^{-15}$. 


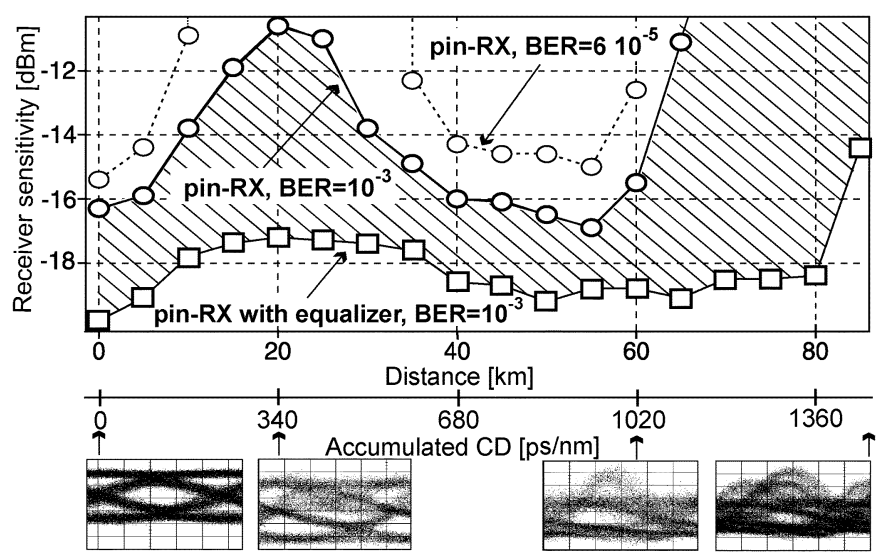

Fig. 9. Receiver sensitivity (solid: $\mathrm{BER}=10^{-3}$, dashed: $\mathrm{BER}=6 \cdot 10^{-5}$ ) versus transmission distance and accumulated chromatic dispersion (CD) for the 1550-nm CWDM laser. Circles: Unequalized receiver. Squares: Equalized receiver. Hatched area: Equalization gain. Optical eye diagrams are shown at various distances.

\section{Chromatic Dispersion, Equalization, AND FEC}

Fig. 9 shows, as a typical example, the receiver sensitivity as a function of the transmission distance for the 1550-nm CWDM channel without (circles) and with (squares) electronic equalization, using an FEC-precoded PRBS (length $2^{31}-1$ ) at 10.664 $\mathrm{Gb} / \mathrm{s}$. Both solid curves were taken at a channel BER of $10^{-3}$ and with enabled FEC to ensure block error correctability. The dotted curve shows sensitivities at $\mathrm{BER}=6 \cdot 10^{-5}$. All curves used the same laser driving conditions, optimized for maximum reach. The improvement in receiver performance beyond $20 \mathrm{~km}$ reflects the effect of self-steepening, caused by the interplay of chromatic dispersion and adiabatic laser chirp, leading to pulse recompression [19], [20]. The effect of equalization, visualized by the hatched area in Fig. 9, is twofold: first, as discussed in Section III, equalization provides a back-to-back sensitivity improvement of almost $4 \mathrm{~dB}$, which compensates for the limited $10-\mathrm{Gb} / \mathrm{s}$ performance of our $2.5-\mathrm{Gb} / \mathrm{s}$ rated DMLs. For the compressed pulses at around $50 \mathrm{~km}$ we find an equalization gain of 2-3 dB. Second, at distances where the pulses are broadened by dispersion (at distances around $20 \mathrm{~km}$ and in excess of $60 \mathrm{~km}$ ), the equalizer gain exceeds $7 \mathrm{~dB}$. Using equalization and FEC, error-free transmission over $85 \mathrm{~km}$ was achieved, corresponding to an accumulated CD of $1445 \mathrm{ps} / \mathrm{nm}$ at $1550 \mathrm{~nm}$. Note from the dotted curve in Fig. 9 that transmission between 15 and $35 \mathrm{~km}$ of standard-dispersion fiber was not possible at a channel BER of $6 \cdot 10^{-5}$ due to severe error flooring. Since this channel BER corresponds to the correction threshold of standard $\operatorname{RS}(255,239)$ FEC (cf. Fig. 8), we conclude that only the combination of electronic equalization and enhanced FEC enables 10-Gb/s CWDM to continuously attain appreciable distances, as needed in flexible metro networks.

It is important to note from Fig. 9 that electronic equalization significantly reduces the variations in chromatic dispersion penalty over distance. In particular, the poor receiver sensitivity around $20 \mathrm{~km}$ is substantially improved. This feature of electronic equalization significantly facilitates system design, as visualized in Fig. 10, showing the measured 1550-nm receiver sensitivities from Fig. 9. Also shown is the signal power evolution as a function of distance (dashed), for a signal launch power

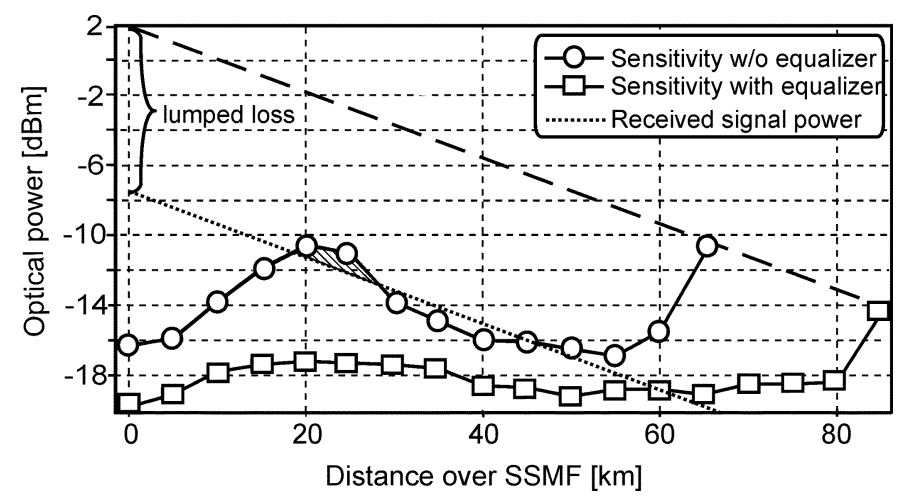

Fig. 10. Receiver sensitivity at $B E R=10^{-3}$ versus transmission distance for the 1550-nm CWDM laser (circles: unequalized receiver; squares: equalized receiver). The dashed line represents the optical power evolution as a function of transmission distance, while the dotted line includes any lumped optical loss and represents the available optical power at the receiver. The hatched area indicates unattainable transmission distances caused by high penalty around $20 \mathrm{~km}$ in the unequalized case.

of $+2 \mathrm{dBm}$ and a fiber loss coefficient of $0.19 \mathrm{~dB} / \mathrm{km}$. Any additional lumped loss, e.g., due to multiplexers, connectors, couplers, or splices, shifts down the dashed curve, resulting in the dotted curve in Fig. 10, representing the available optical power at the receiver as a function of transmission distance. System margins may also be added to further lower the dotted curve. The intersection of the available power with the receiver sensitivity then determines the maximum transmission distance. Typically, the solution to finding the maximum transmission distance is unique, i.e., the available power and the receiver sensitivity intersect only once. However, if the receiver sensitivity exhibits nonmonotonic behavior as a function of distance, as found for the unequalized receiver (circles), more than one intersection may occur, which in the equalizer-free example of Fig. 10 excludes transmission distances between 10 and $30 \mathrm{~km}$ (hatched area in Fig. 10), but otherwise enables distances of up to $45 \mathrm{~km}$. Such a situation significantly complicates system design, unless one is willing to accept the lowest-distance intersection as the maximum allowed transmission distance. In general, the criterion for the sensitivity penalty to yield unattainable transmission distances (and thereby complicate system design) reads

$$
-\frac{\partial P_{\text {sens }}}{\partial z}>\alpha
$$

where $P_{\text {sens }}$ denotes receiver sensitivity in decibels, $z$ is the coordinate in propagation direction in kilometers, and $\alpha$ stands for the fiber attenuation coefficient in $\mathrm{dB} / \mathrm{km}$. As evident from Figs. 9 and 10, electronic equalization substantially reduces the variations in receiver sensitivity, and thus facilitates system design.

\section{IN-BAND CROSSTALK AND FEC}

Fully bidirectional optical transmission systems, making the most efficient use of installed fiber infrastructure, have been frequently discussed, both for amplified multispan transmission and for single-span access scenarios [21], [22]. So far, however, their deployment has been prevented by beat noise due to in-band crosstalk, arising from connector reflections and Rayleigh backscatter. This places severe restrictions on 


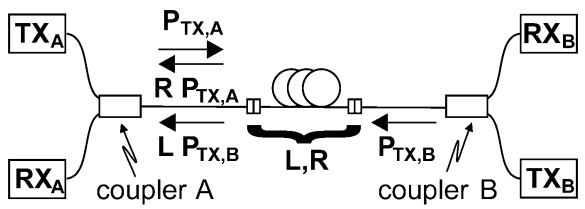

Fig. 11. Fully bidirectional fiber communication system.

the tolerable link loss, and thus on the achievable transmission distances. Fig. 11 illustrates this limitation: Transmitter $T X_{B}$ sends information to be received by receiver $R X_{A}$. The signal power after coupler $B$ is denoted $P_{T X, B}$. Due to (lumped and distributed) span loss $L$, the signal power just before coupler $A$ is $L P_{T X, B}$. At the same time, a reflected power $R P_{T X, A}$ will be present at that point, caused by any (lumped and distributed) reflections $R$ of the counter-propagating signal within the span, as shown in the figure. The ratio of signal power to interferer power at the receiver [signal-to-interference ratio (SIR)] can then be expressed in $\mathrm{dB}$ as [22]

$$
\mathrm{SIR}_{[d B]}=L_{[d B]}-R_{[d B]}-\Delta P_{[d B]}
$$

where $\Delta P=P_{T X, A} / P_{T X, B}$ is the power divergence between same-wavelength transmitters. Typically, $R$ is around $-32 \mathrm{~dB}$ [22], and $\mathrm{SIR}>25 \mathrm{~dB}$ is required for less than 1-dB power penalty at a BER of $10^{-9}$, a pin-type photoreceiver, and non return-to-zero (NRZ) modulation [22]-[26]. If the interferers are due to a single discrete reflection (e.g., a connector) rather than due to multiple reflections (e.g., Rayleigh backscatter), the tolerable SIR can be somewhat lower [24]. Allowing for a transmitter power divergence of $5 \mathrm{~dB}$, (2) predicts a maximum tolerable span loss of $2 \mathrm{~dB}$, which translates into a transmission distance of some $10 \mathrm{~km}$ at $1550 \mathrm{~nm}$. (Note that the span loss $L$ excludes CWDM multiplexers and couplers.)

Using FEC, the requirements on SIR can be significantly relaxed, and the bidirectional transmission distance can be extended. This beneficial aspect of FEC [27]-[29] owes to the fact that in-band crosstalk, like many other optical signal degradations [30], [31], leads to reduced sensitivity penalties at poor channel BER (where FEC operates) as compared to operation at low channel BER (uncoded systems): At low BER, e.g., at $10^{-9}$, it only takes a few highly distorted bits within a bit pattern to significantly affect the overall BER of the pattern or to even produce a noticeable error floor, while at poor BER, e.g., at $10^{-3}$, the incremental contribution of a few distorted bits to the overall BER of the pattern is negligible. For the case of in-band crosstalk, the BER-dependence of power penalties is visualized in Fig. 12(a), showing the BER as a function of received signal power for a range of different SIRs, measured with a $10-\mathrm{Gb} / \mathrm{s}$ rated DML at $1550 \mathrm{~nm}$ [eye diagram of Fig. 12(b), 7.5-dB extinction] according to the setup in Fig. 12(c). In the measurements, we used two optical attenuators to set SIR and received signal power, respectively. A polarization controller was used to achieve worst-case interference conditions by co-polarizing signal and interferer, which were decorrelated by a $10-\mathrm{km}$ long spool of nonzero dispersion shifted fiber (NZDF). We used our pin-receiver frontend, without equalization but with enabled FEC.
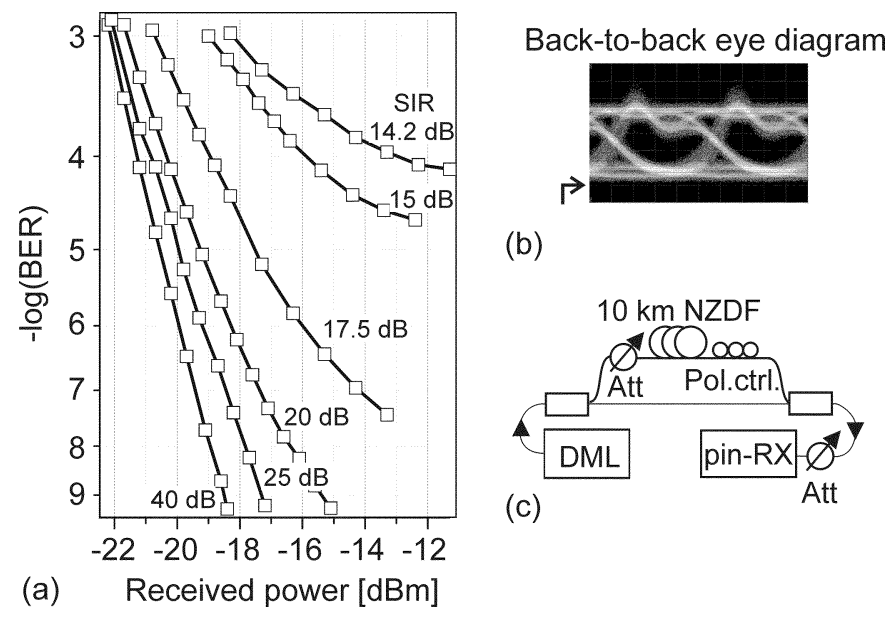

(b)

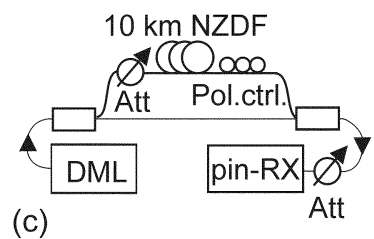

Fig. 12. (a) Measured BER as a function of received signal power for different signal-to-interferer ratios (SIR) using a 10-Gb/s rated DML at 1550 nm. (b) Back-to-back eye diagram of the 10-Gb/s laser (without interferers). (c) Measurement setup, incorporating two attenuators to set SIR and received power, a polarization controller to align signal and interferer, and $10 \mathrm{~km}$ of nonzero dispersion shifted fiber (NZDF) to decorrelate signal and interferer.

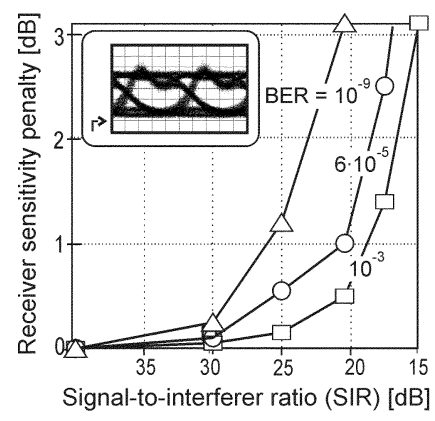

(a) 10-Gb/s DML, 7.5-dB extinction

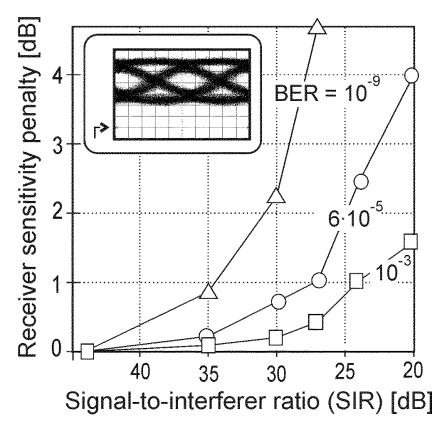

(b) 2.5-Gb/s DML, $\sim 3-\mathrm{dB}$ extinction
Fig. 13. 10-Gb/s receiver sensitivity penalties versus SIR at different target BERs, measured at $1550 \mathrm{~nm}$ using the setup of Fig. 12(c). (a) 10-Gb/s rated DML with $\sim 7.5-\mathrm{dB}$ extinction. (b) $2.5-\mathrm{Gb} / \mathrm{s}$ rated $\mathrm{DML}$ with $\sim 3-\mathrm{dB}$ extinction.

Fig. 13 shows the BER-dependent sensitivity penalties as a function of SIR, both for the 10-Gb/s rated DML (a) and for the $2.5-\mathrm{Gb} / \mathrm{s}$ rated DML (b) at $1550 \mathrm{~nm}$. Both DMLs exhibit a significantly increased tolerance to in-band crosstalk at FEC channel error ratios [27]-[29]. In Fig. 13(a), the 1-dB penalty is pushed from 26 to $19 \mathrm{~dB}$ when going from $\mathrm{BER}=10^{-9}$ to $10^{-3}$, which in the above example increases the tolerable span attenuation to $8 \mathrm{~dB}$ (or $40 \mathrm{~km}$ at $1550 \mathrm{~nm}$ ). Since the data extinction ratio has a strong impact on the beat-noise induced penalties caused by in-band crosstalk [24], the poor extinction $(\sim 3 \mathrm{~dB})$ of the $2.5-\mathrm{Gb} / \mathrm{s}$ rated DML operated at $10 \mathrm{~Gb} / \mathrm{s}$ [Fig. 13(b)] artificially reduces the allowable SIR, and is not too representative of realistically deployable $10-\mathrm{Gb} / \mathrm{s}$ systems.

\section{CWDM System SETUPS AND TRANSMISSION RESUlTS}

Using electronic equalization and FEC as our key enabling technologies, we set up the two CWDM systems depicted in Fig. 14: All 16 uncooled DMLs were driven with an FEC-precoded PRBS (length $2^{31}-1$ ) using nonreturn-to-zero modulation at $10.664 \mathrm{~Gb} / \mathrm{s}$. The average laser power varied 


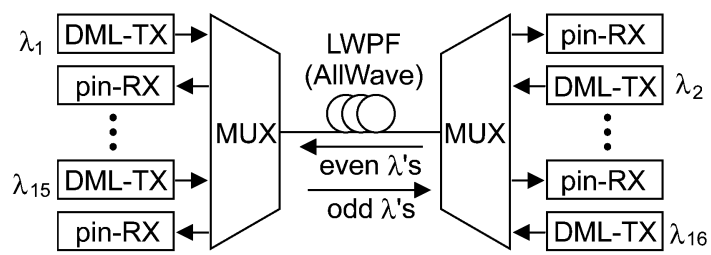

(a)

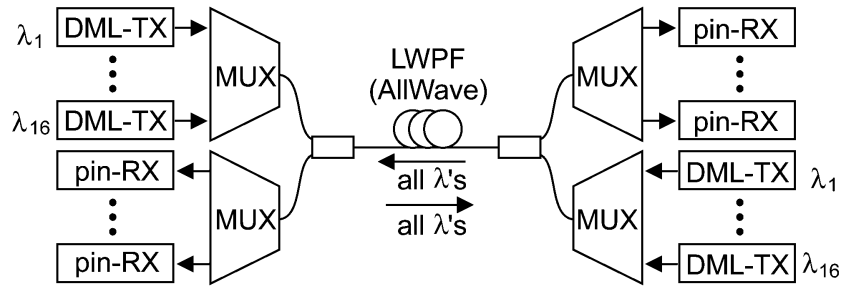

(b)

Fig. 14. Two 16-channel CWDM systems over standard-dispersion fiber. (a) The eight even wavelengths propagate West, and the eight odd wavelengths propagate East. (b) All 16 wavelengths propagate in both directions over a single optical fiber.
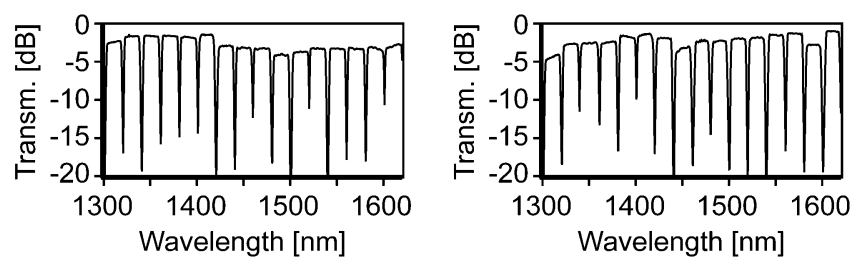

Fig. 15. Optical power transmission of the two 16-channel CWDM thin-film multiplexers.

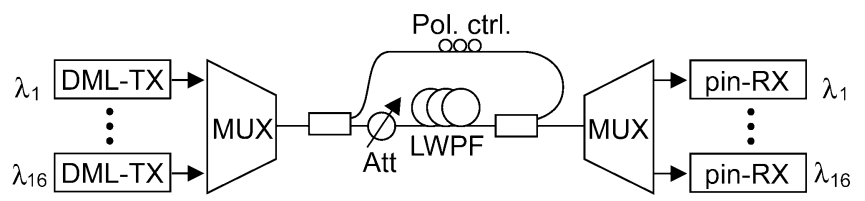

Fig. 16. Experimental setup to generate backward traffic and emulate the worst-case scenario for the fully bidirectional system of Fig. 14(b).

from 5 to $10 \mathrm{dBm}$ among the channels. In order to simultaneously drive all 16 lasers, we used electrical amplification and a subsequent electrical power splitting network. Commercially available thin-film CWDM multiplexers with channel-dependent insertion losses between 1 and $4 \mathrm{~dB}$ were used to combine the CWDM sources. The transfer characteristics of the thin film multiplexers are shown in Fig. 15. An additional 2-3 $\mathrm{dB}$ of overall connector loss was distributed throughout the systems, consisting of combinations of connectorized fiber spools to achieve different transmission lengths. In the setup sketched in Fig. 14(a), the 16 channels were partitioned, with 8 channels propagating in each direction along a standard-dispersion LWPF, whose characteristics are given in Fig. 1. In the $2 \times 16$ channel system of Fig. 14(b) we used fully bidirectional transmission, with all 16 channels propagating in each direction along the fiber. Because only one set of 16 CWDM channels was available to experimentally test the system of Fig. 14(b), we used the setup of Fig. 16, where we split off a fraction of the CWDM signal by means of a broadband optical coupler prior
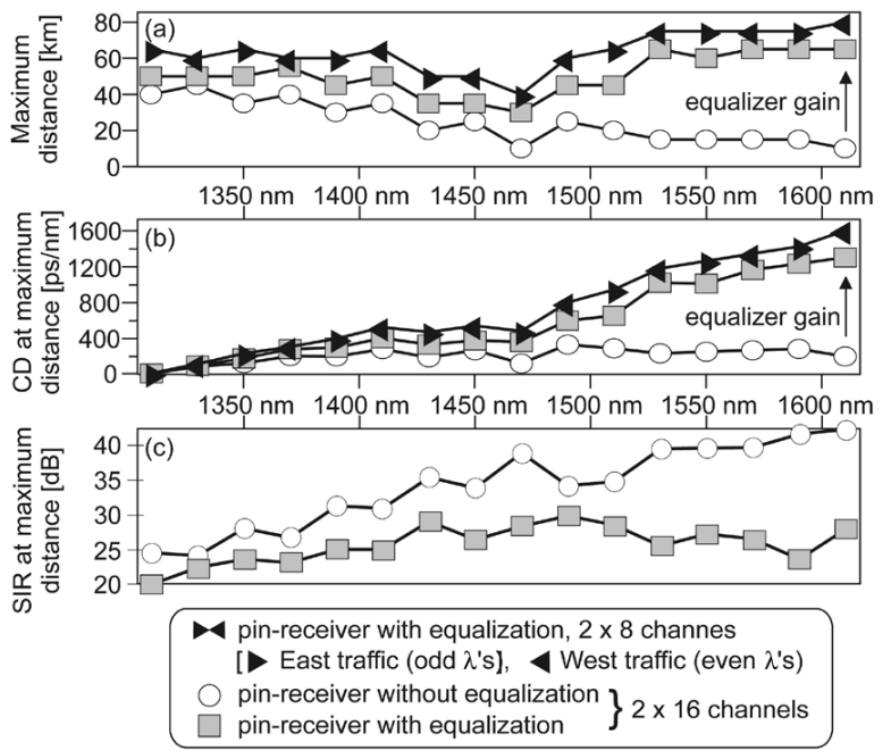

Fig. 17. (a) Maximum transmission distance for all CWDM channels. (b) Chromatic dispersion at maximum distance shown in (a). (c) SIR measured in the fully bidirectional experiment with lower power for the backward traffic than for the signal Black triangles: $2 \times 8$-channel experiment using equalization Gray squares: $2 \times 16$-channel experiment using equalization Open circles: $2 \times 16$-channel experiment w/o equalization.

to entering the span. At the receiver side, we used a second optical coupler to feed in the counter-propagating channels. Appropriate selection of the couplers in combination with an attenuator at the input to the span set the ratio of signal powers entering the span from each side. A polarization controller was used to align, for each CWDM wavelength separately, the reflections from backward (West) traffic with the signal (East traffic) for maximum impairment. Although we used various types of angled and straight fiber connectors, the dominant source of in-band crosstalk was Rayleigh backscatter from backward traffic.

Fig. 17(a) (black triangles) shows the results of the transmission experiment according to Fig. 14(a), using electronic equalization and FEC; the triangles point in the direction of traffic flow (East and West traffic). The significant performance variations among channels are typical for nonselected DMLs (cf. Fig. 6). In the long-wavelength region (high dispersion but low loss), the equalizer proves particularly valuable, enabling transmission distances in excess of $40 \mathrm{~km}$ for all channels and up to $80 \mathrm{~km}$ for the best-performing channel; more than $50 \%$ of all channels go beyond $65 \mathrm{~km}$.

Also shown in Fig. 17 are the results for the setup of Figs. 14(b) and 16. Electronic equalization is seen to boost transmission distances from $10 \mathrm{~km}$ up to $65 \mathrm{~km}$. As is evident from Fig. 14(b), the unequalized receiver only supports $\sim 300 \mathrm{ps} / \mathrm{nm}$ of $\mathrm{CD}$, while the equalizer allows up to 1600 $\mathrm{ps} / \mathrm{nm}$. Note that these fully bidirectional transmission results were mistakenly labeled as "equal launch power in both directions" in [10], owing to a measurement error which we only became aware of after the publication of [10] and which we want to correct at this point: The curves for the $2 \times 16$-channel bidirectional system shown in [10] as well as in Fig. 17 refer to a launched backward traffic that was lower than the signal 
traffic by $5 \mathrm{~dB}$ (lower wavelengths) to $8 \mathrm{~dB}$ (higher wavelengths). Therefore, the measured SIR corresponding to the fully bidirectional transmission distances of Fig. 17(a) was always better than $20 \mathrm{~dB}$ [see Fig. 17(c)], and significant limitations from in-band crosstalk did not surface. Thus, the gray squares in Fig. 17 follow closely the black triangles, offset by a near-constant penalty due to the additional loss of the two couplers in Fig. 16. Since publication of [10], we have repeated the fully bidirectional experiment for the equalizing receiver by further attenuating the signal in order to launch equal powers for both traffic directions, and found that we were limited by in-band crosstalk to a transmission distance of 30 $\mathrm{km}$ for the lower-wavelength channels (with SIRs between 16 and $20 \mathrm{~dB}$ ), and to $10 \mathrm{~km}$ for the highest-wavelength channels. Note, however, that this severe crosstalk limitation for the long-wavelength channels is solely due to the poor extinction of the 2.5-Gb/s rated DMLs (cf. Figs. 5 and 13). To prove this point, we carried out the same fully bidirectional experiment with a 10-Gb/s rated DML at $1550 \mathrm{~nm}$, showing an extinction of $\sim 7.5 \mathrm{~dB}$, and obtained a transmission distance of $60 \mathrm{~km}$ $(\mathrm{SIR}=17.6 \mathrm{~dB})$ for equal launch powers in both traffic directions. Thus, despite having demonstrated the key enablers for bidirectional CWDM transport, we have to conclude that a demonstration of full bidirectionality, bridging significant distances over the entire CWDM band, has to await the availability of proper laser sources.

\section{CONCLUSION}

We have experimentally demonstrated that advanced electronic equalization and FEC can be used to upgrade full-band CWDM systems from $2.5 \mathrm{~Gb} / \mathrm{s}$ per-channel to $10 \mathrm{~Gb} / \mathrm{s}$ while maintaining appreciable transmission distances over standard-dispersion fiber. Due to the lack of a full set of CWDM sources rated for $10-\mathrm{Gb} / \mathrm{s}$, we demonstrated full-spectrum (1310 to $1610 \mathrm{~nm}$ ) 10-Gb/s CWDM transmission over standard-dispersion fiber using uncooled, directly modulated lasers specified for $2.5 \mathrm{~Gb} / \mathrm{s}$. The lasers were not preselected. All 16 CWDM channels were transmitted over more than $40 \mathrm{~km}$, yielding a capacity-times-distance product of $6.4 \mathrm{~Tb} / \mathrm{s} / \mathrm{km}$. The longest CWDM transmission distance $(80 \mathrm{~km})$ was achieved at $1610 \mathrm{~nm}$, equivalent to $1600 \mathrm{ps} / \mathrm{nm}$ of chromatic dispersion. In addition, we showed how FEC can be used to enhance the tolerance to in-band crosstalk, which poses a major limitation to fully bidirectional systems. Using a $10-\mathrm{Gb} / \mathrm{s}$ rated laser at $1550 \mathrm{~nm}$, we demonstrated the capability of single-wavelength bidirectional transmission over $60 \mathrm{~km}$ of standard-dispersion fiber.

\section{REFERENCES}

[1] S. K. Das et al., " $40 \mathrm{~Gb} / \mathrm{s}(16 \times 2.5-\mathrm{Gb} / \mathrm{s})$ full spectrum coarse WDM transmission over $75 \mathrm{~km}$ low water peak fiber for low-cost metro and cable-TV applications," in NFOEC 2002, Dallas, TX, pp. 881-887.

[2] H. J. Thiele et al., "Capacity-enhanced coarse WDM transmission using $10 \mathrm{Gbit} / \mathrm{s}$ sources and DWDM overlay," Electron. Lett., vol. 39, no. 17, 2003.

[3] Sogawa et al., "Study on full-spectrum directly modulated CWDM transmission of $10 \mathrm{~Gb} / \mathrm{s}$ per channel over water-peak-suppressed nonzero dispersion shifted fiber," in ECOC'02 8.2.1, 2002.
[4] G. Sakaino et al., "Transmission characteristics of uncooled and directly modulated $1.3 \mu \mathrm{m}$ distributed feedback laser diode for serial 10 Gigabit Ethernet," in 17th Int. Semiconductor Laser Conf., vol. 89, 2000.

[5] G. Berry et al., " $100^{\circ} \mathrm{C}, 10-\mathrm{Gb} / \mathrm{s}$ directly modulated InGaAsP DFB lasers for uncooled Ethernet applications," in $O F C^{\prime} 02$, 2002, Paper ThF1, pp. 415-416.

[6] K. Sato et al., "Direct modulation of a distributed feedback laser for 40 $\mathrm{Gb} / \mathrm{s}$ very-short-reach optical links," in OFC'02, 2002, Paper ThF2.

[7] B. Wedding et al., "43 Gbit/s transmission over $210 \mathrm{~km} \mathrm{SMF}$ with a directly modulated laser diode," in Proc. ECOC'03, 2003, Paper Mo4.3.7, pp. 98-99.

[8] H. J. Thiele et al., " $16 \times 10-\mathrm{Gb} / \mathrm{s}$ CWDM transmission over $40 \mathrm{~km}$ using uncooled, directly modulated lasers rated for $2.5 \mathrm{~Gb} / \mathrm{s}$," in $O E C C^{\prime} 03$, 2003, Paper PD 4.

[9] H. S. Chung et al., "Directly modulated CWDM/DWDM system using negative dispersion fiber for metro network application," in Proc. OFC'04, 2004, Paper WG5.

[10] P. J. Winzer et al., "Electronic equalization and FEC enable bidirectional CWDM capacities of $9.6 \mathrm{~Tb} / \mathrm{s}-\mathrm{km}$," in Proc. OFC'04, 2004, Paper PDP7.

[11] Tomkos et al., "10-Gb/s transmission of $1.55-\mu \mathrm{m}$ directly modulated signal over $100 \mathrm{~km}$ of negative dispersion fiber," Photon. Technol. Lett., vol. 13, pp. 735-735, 2001.

[12] M. D. Feuer et al., "Electronic dispersion compensation for a $10-\mathrm{Gb} / \mathrm{s}$ link using a directly modulated laser," Photon. Technol. Lett., vol. 15 , pp. 1788-1788, 2003.

[13] C. R. S. Fludger et al., "Electronic equalization for low cost $10 \mathrm{Gbit} / \mathrm{s}$ directly modulated systems," in Proc. OFC'04, 2004, Paper WM7.

[14] D. Castagnozzi, "Digital signal processing and electronic equalization (EE) of ISI," in Proc. OFC'04, 2004, Paper WM6.

[15] J. G. Proakis, Digital Communications. New York: McGraw-Hill, 1995.

[16] J. Winters et al., "Electrical signal processing techniques in long-haul fiber-optic systems," IEEE Trans. Commun., vol. 38, pp. 1439-1439, 1987.

[17] H. Bulow et al., "Electronic PMD mitigation-From linear equalization to maximum likelihood detection," in Proc. OFC'01, 2001, Paper WAA3-3.

[18] H. F. Haunstein et al., "Design of near optimum electrical equalizers for optical transmission in the presence of PMD," in Proc. OFC'01, 2001, Paper WAA4-1.

[19] P. J. Corvini et al., "Computer simulation of high-bit-rate optical fiber transmission using single-frequency lasers," J. Lightw. Technol., vol. 5, pp. 1591-1591, 1987.

[20] S. Mohrdiek et al., "10-Gb/s standard fiber transmission using directly modulated 1.55- $\mu \mathrm{m}$ quantum-well DFB lasers," Photon. Technol. Lett., vol. 7, pp. 1357-1359, 1995.

[21] S. Radic et al., "Dense interleaved bidirectional transmission over $5 \times 80 \mathrm{~km}$ of nonzero dispersion-shifted fiber," Photon. Technol. Lett., vol. 14, pp. 218-220, 2002.

[22] S. K. Das et al., "Beat interference penalty in optical duplex transmission," J. Lightw. Technol., vol. 20, pp. 213-213, 2002.

[23] E. L. Goldstein et al., "Performance implications of component crosstalk in transparent lightwave networks," Photon. Technol. Lett., vol. 6, pp. 657-660, 1994.

[24] C. J. Rasmussen et al., "Theoretical and experimental studies of the influence of the number of crosstalk signals on the penalty cuased by incoherent optical crosstalk," in Proc. OFC'99, 1999, Paper TuR5, pp. 258-260.

[25] F. Liu et al., "Experimental verification of a new model describing the influence of incomplete signal extinction ratio on the sensitivity degradation due to multiple interferometric crosstalk," Photon. Technol. Lett. vol. 11, pp. 137-139, 1999.

[26] C. R. S. Fludger et al., "Impact of MPI and modulation format on transmission systems employing distributed Raman amplification," Electron. Lett., vol. 37, pp. 970-972, 2001.

[27] S. Chandrasekhar et al., "Performance of FEC in the presence of in-band crosstalk," in $O F C^{\prime} 02$, vol. 288, 2002.

[28] S. Radic et al., "Forward error correction performance in the presence of Rayleigh-dominated transmission noise," Photon. Technol. Lett., vol. 15, pp. 326-328, 2003.

[29] V. Kaman et al., "Mitigation of optical crosstalk penalty in photonic cross-connects using forward error correction," Electron. Lett., vol. 39, pp. 678-679, 2003.

[30] P. J. Winzer et al., "Coherent crosstalk in ultra-dense WDM systems," in Proc. ECOC'04, 2004.

[31] — "Dispersion-tolerant optical communication systems," in Proc. ECOC'04, 2004 


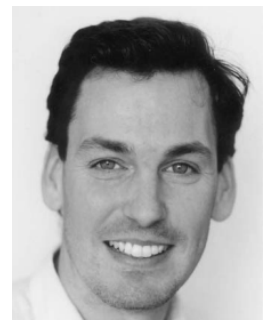

P. J. Winzer (S'93-M'03) received the M.S. and Ph.D. degrees in electrical engineering/communications engineering from Vienna University of Technology, Vienna, Austria, in 1996 and 1998, respectively.

His work, largely supported by the European Space Agency (ESA), was related to the analysis and modeling of space-borne Doppler wind lidar, and optical communication systems, with an emphasis on optical modulation formats and high-sensitivity receivers employing coherent as well as direct detection. Following his time as a research and teaching assistant at the Vienna University of Technology, he joined Lucent Technologies, Bell Laboratories, Holmdel, NJ, in 2000, where he has since been working on fiber-optic communications, including Raman amplification, $40-\mathrm{Gb} / \mathrm{s}$ optical transmitter and receiver design, optical and electronic equalization, and advanced optical modulation formats, as well as on novel architectures for data networking. He has authored and coauthored over 100 journal and conference papers and holds several patents on optical communications and lidar.

Dr. Winzer is a Member of the Optical Society of America (OSA).

F. Fidler, photograph and biography not available at the time of publication.

M. J. Matthews, photograph and biography not available at the time of publication.

L. E. Nelson, photograph and biography not available at the time of publication.

H. J. Thiele, photograph and biography not available at the time of publication.

J. H. Sinsky, photograph and biography not available at the time of publication.

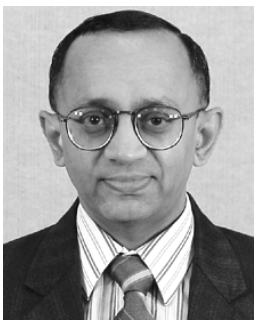

S. Chandrasekhar (M'90-SM'00-F'01) received the B.Sc., M.Sc., and Ph.D. degrees in physics from the University of Bombay, Bombay, India, in 1973, 1975 , and 1985 , respectively.

He joined the Tata Institute of Fundamental Research, Bombay, India, as a Research Scholar in 1975 and then became a Research Associate in 1979. He had been engaged in research on $\mathrm{Si}-\mathrm{SiO}_{2}$ interface studies, complementary metal-oxide-semiconductor integrated circuits, ion implantation, charge-coupled devices, and laser recrystallization. In 1986, he joined AT\&T Bell Laboratories (now called Lucent Technologies, Bell Laboratories), Crawford Hill Laboratory, Holmdel, NJ, as a Postdoctoral Member of Technical Staff. Since then, he has been working on III-V compound semiconductor devices for optoelectronic applications, primarily waveguides, photodetectors, heterojunction phototransistors, and heterojunction bipolar transitors (HBTs). He became a permanent Member of Technical Staff in May 1992. He has been engaged in high-speed optoelectronic integrated circuits (OEICs), integrating $\mathrm{p}$-i-n photodetectors and laser diodes with HBTs, for long-wavelength optical communications. Since January 1999, he has been responsible for forward-looking research in wavelength-division-multiplexing (WDM) optical networking. He has set up an optical networking testbed for investigation of optical networks comprising add/drop multiplexers, optical cross connects, and wavelength-selective switching. His current interests include $40-\mathrm{Gb} / \mathrm{s}$ transport and networking, modulation formats, and electronic signal processing at the receiver. He is currently a Distinguished Member of Technical Staff. He holds 13 U.S. patents.

Dr. Chandrasekhar is a Member of the IEEE Lasers \& Electro-Optics Society (LEOS). He has been Associate Editor of IEEE PHOTONICS TECHNOLOGY LETTERS since 1998. He has been member of the technical program committes of the International Electron Devices Meeting (IEDM), the Device Research Conference (DRC), and the Optical Fiber Communications (OFC) conferences. $\mathrm{He}$ was awarded the IEEE LEOS Engineering Achievement for 2000 jointly with two other awardees for his contribution to OEIC photoreceivers and the Optical Society of America (OSA) Engineering Excellence Award for 2004 for his contributions to OEICs and WDM systems research.

M. Winter, photograph and biography not available at the time of publication.

D. Castagnozzi, photograph and biography not available at the time of publication.

L. W. Stulz, photograph and biography not available at the time of publication.

H. Buhl, photograph and biography not available at the time of publication. 